\title{
INFRARED RADIATION FROM EVOLVED STARS
}

\author{
William K. Rose \\ Astronomy Program \\ University of Maryland \\ College Park, MD 20742 USA
}

\begin{abstract}
OH/IR stars and carbon stars are examples of evolved stars known to lose mass rapidly. In a recent paper (Rose 1987) we have described calculations of infrared continuum radiation from dust in stellar winds from red giants. We compare calculated radiative models with recent infrared measurements and estimate the total contribution of evolved stars to the mid-infrared background radiation observed from the Milky Way and M31.
\end{abstract}

\section{INFRARED CONTINUUM RADIATION FROM DUST}

In a recent paper (Rose 1987, Paper I) we have described calculations of infrared continuum radiation from dust embedded in outflowing gas from red giants. These calculations assume spherically symmetric mass loss. The numerical solutions described in Paper I depend on stellar luminosity, effective temperature of central star, mass loss rate, radial distance at which grain formation occurs, mass fraction in grains, and characteristic grain mass. In addition the fraction of absorbed photons of frequency $v$ per unit area must also be specified. Grain temperatures and emissivities at various distances from the central star are determined by the condition of radiative equilibrium between grain emission and absorption of radiation from the central star and surrounding circumstellar grains. Two types of mass loss, namely constant mass loss and mass loss in ejected shells, were considered in the calculations.

A comparison between IRAS observations of Mira variables (Olnon et al. 1984) and models described in Paper I showed that calculated models were restricted to a much smaller region of the color-color diagram defined by $\log v F_{v}(25)-\log v F_{v}(12)$ vs. $\log v F_{v}(60)-$ $\log \vee F_{v}(25)$. It was suggested in Paper I that the infrared colors of most observed Mira variables were primarily the result of emission from dust close to the central star and not a steady stellar wind. IRAS observations can be interpreted to imply that steady-state mass loss rates are less than $10^{-6} \mathrm{M}_{\odot \mathrm{yr}^{-1}}$ for most Mira variables. This result is consistent with mass loss rates of $10^{-7}-10^{-6} M \odot \mathrm{yr}^{-1}$ estimated by Knapp and Morris (1985). A similar comparison in the same color-color diagram between OH/IR stars observed by Olnon et al. (1984) and calculated models (Paper I) with higher mass loss rates gave agreement with observations for $M \geq 10^{-5} M_{\odot} \mathrm{yr}^{-1}$. Recently Bedijn (1987) has compared theoretical models with a sample of Mira variables and variable $\mathrm{OH} / \mathrm{IR}$ stars and shown that they occupy a relatively narrow continuous region in the color-color diagram. These results are consistent with our calculated spectra (Paper I) for red giants with steady outflow and mass ejection in shells. The corresponding positions of nonvariable OH/IR stars in the color-color diagram indicate that rapid mass ejection has occurred recently $\left(<10^{3} \mathrm{yr}\right)$. 


\section{MID-INFRARED RADIATION FROM THE MILKY WAY, M31, AND THE MAGELLANIC CLOUD}

At near infrared wavelengths (i.e., 1-3 $\mu \mathrm{m}$ ), background infrared radiation from the Milky Way and M31 can be explained as radiation from the photospheres of $K$ and $\mathbf{M}$ giants. However, photospheric emission is insufficient to account for the observed mid-infrared $(10-30 \mu \mathrm{m})$ radiation. Dust embedded in $\mathrm{HI}$ regions and molecular clouds is the source of far infrared radiation $(60-100 \mu \mathrm{m})$. The color temperature of far infrared radiation is too high $(T \simeq 40 \mathrm{~K})$ for molecular clouds to contribute appreciably at relatively short far-infrared wavelengths but is consistent with heating of $\mathrm{HI}$ regions caused by $\mathrm{O}$ and $\mathrm{B}$ stars.

Infrared fluxes from the Milky Way and M31 decline more rapidly between $10^{2}$ and $60 \mu \mathrm{m}$ than in either the Large Magellanic Cloud (LMC) or Small Magellanic Cloud (SMC), which are known to have appreciably less dust than either the Milky Way or M31. The Magellanic Clouds have similar infrared spectra with a decline in flux between 25 and $12 \mu \mathrm{m}$, whereas the spectra of both the Milky Way and M31 show excess radiation at mid-infrared wavelengths. The percentage of emission from cool gas is higher in the spiral galaxies than in the Magellanic Clouds. Differences in infrared spectra probably indicate that mid-infrared radiation from evolved stars (principally red giants) is relatively greater in the Milky Way and M31 than in the Magellanic Clouds. Lower infrared fluxes from evolved stars in the LMC and SMC are probably caused by lower abundances of heavy elements and correspondingly lower abundances of dust. The temperature of dust within the Magellanic Clouds is higher than in spiral galaxies because the ultraviolet radiation field is stronger, and in addition spiral galaxies have larger amounts of cold dust within molecular clouds.

Approximately one white dwarf is formed each year within the Galaxy. Most intermediate mass stars lose sufficient amounts of mass on the asymptotic giant branch to evolve into white dwarfs. We assume that intermediate mass stars form one white dwarf every two years and that the average total mass loss when the star is either an OH/IR star or carbon star is $2 M_{\odot}$. There is considerable variation in mass loss rate on the asymptotic giant branch. The mass loss rates of Mira variables are usually $\leq 10^{-6} M \odot \mathrm{yr}^{-1}$, whereas those of $\mathrm{OH} / \mathrm{IR}$ stars and carbon stars, which account for most of the mid-infrared radiation from evolved stars, are $\geq 10^{-5} M \odot \mathrm{yr}^{-1}$. If we assume an average mass loss rate of $2 \times 10^{-5} \mathrm{M}_{\odot} \mathrm{yr}^{-1}$, then the evolutionary time required to lose $2 M_{\odot}$ becomes $\simeq 10^{5}$ yr and, consequently, there are approximately $5 \times 10^{4}$ such stars in the Milky Way, compared with approximately $10^{4}$ planetary nebulae. Our estimates of total mass loss include planetary nebulae ejected by intermediate mass stars. Moreover, they are consistent with the estimate of $6 \times 10^{4} \mathrm{OH} / \mathrm{IR}$ stars given by Cox, Krugel, and Mezger (1986).

Because OH/IR stars and carbon stars have mass loss rates $\geq 10^{-5} M \odot \mathrm{yr}^{-1}$, the outflowing gas is (nearly) optically thick to photospheric emission, and therefore most stellar radiation is re-emitted at mid-infrared wavelengths. For an assumed average luminosity $1-2 \times 10^{4} L_{\odot}$, the predicted mid-infrared luminosity becomes $=0.5-1 \times 10^{9} L_{\odot}$. We estimate that the total mid-infrared radiation emitted by low mass stars and lower luminosity intermediate mass stars that are Mira variables is approximately equal to the above estimate. We regard $10^{4} L_{\odot}$ as a lower limit for the average luminosity of OH/IR stars, and therefore the estimated mid-infrared radiation from the Milky Way becomes $1-2 \times 10^{9} L_{O}$, which is in agreement with value of $\simeq 2 \times 10^{9} L \odot$ given by Cox, Krugel, and Mezger (1986) for the total hot dust luminosity.

In Figure 1 the infrared spectra of the Milky Way (Cox, Krugel, and Mezger 1986) and M31 (Walterbos and Schwering 1987) are shown with the calculated infrared spectra of a red giant with $L=2 \times 10^{4} L_{\odot}$ and $M=3 \times 10^{-5} M \odot \mathrm{yr}^{-1}$ and a red giant that has undergone rapid mass loss. The infrared spectra of the Milky Way and M31 are similar; however, infrared fluxes from M31 are about a factor of 2 lower, and infrared emission caused by $\mathrm{O}$ and $\mathrm{B}$ stars in star-forming regions is much lower. It follows that the rate of formation of massive 
stars is appreciably higher in the Milky Way than in M31 and that the intermediate mass star formation rate is approximately a factor of 2 higher in the Milky Way.

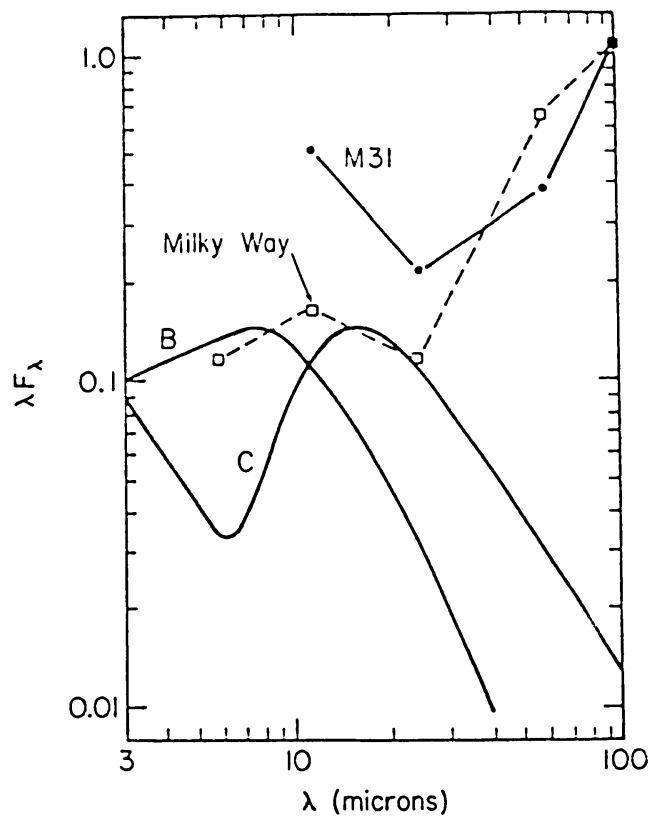

Figure 1. $\lambda F_{\lambda}$ values of the Milky Way and M31 are compared in arbitrary units with those of a red giant with $L=2 \times 10^{4} L_{\odot} M=3 \times 10^{-5} M \odot \mathrm{yr}^{-1}(\mathrm{~B})$, and a red giant of similar luminosity whose mass loss rate had been $M=3 \times 10^{-4} M \odot \mathrm{yr}^{-1}$ (C). The Milky Way and M31 data points are from Cox, Krugel, and Mezger (1986) and Walterbos and Schwering (1987).

An alternative explanation for the origin of mid-infrared radiation is radiation from very small dust grains heated to relatively high temperatures (i.e., $\sim 250-500 \mathrm{~K}$ ). This explanation has been favored for M31 by Walterbos and Schwering (1987) because there is a correlation between optical dust lanes and $12 \mu \mathrm{m}$ emission. In addition, the intensity distributions of 12 and $25 \mu \mathrm{m}$ maps of M31 are similar to those at 60 and $100 \mu \mathrm{m}$. These correlations do not contradict our explanation for mid-infrared radiation because intermediate mass stars are the major contributors. The main sequence lifetime of a $5 \mathrm{M} \odot$ star is approximately $10^{8} \mathrm{yr}$.

\section{REFERENCES}

Bedijn, P. J. 1987, Astron. Astrophys., 186, 136.

Cox, P., Krugel, E., and Mezger, P. G. 1986, Astron. Astrophys., 155, 380.

Knapp, G. R., and Morris, M. 1985, Astrophys. J., 292, 640.

Olnon, F. M., Baud, B., Habing, H. J., DeJong, T., Harris, S., and Pottasch, S. R. 1984, Astrophys. J. (Letters), 278, L41.

Rose, W. K. 1987, Astrophys. J., 312, 284 (Paper I).

Walterbos, R. A. M., and Schwering, P. B. W. 1987, Astron. Astrophys., 180, 27. 